Research Article

\title{
The Asymptotic Stability of the Generalized 3D Navier-Stokes Equations
}

\author{
Wen-Juan Wang and Yan Jia \\ School of Mathematical Sciences, Anhui University, Hefei 230601, China \\ Correspondence should be addressed to Yan Jia; jyan70@163.com
}

Received 8 July 2013; Revised 30 September 2013; Accepted 21 October 2013

Academic Editor: Nicola Guglielmi

Copyright (C) 2013 W.-J. Wang and Y. Jia. This is an open access article distributed under the Creative Commons Attribution License, which permits unrestricted use, distribution, and reproduction in any medium, provided the original work is properly cited.

We study the stability issue of the generalized 3D Navier-Stokes equations. It is shown that if the weak solution $u$ of the NavierStokes equations lies in the regular class $\nabla u \in L^{p}\left(0, \infty ; B_{q, \infty}^{0}\left(\mathbb{R}^{3}\right)\right),(2 \alpha / p)+(3 / q)=2 \alpha, 2<q<\infty, 0<\alpha<1$, then every weak solution $v(x, t)$ of the perturbed system converges asymptotically to $u(x, t)$ as $\|v(t)-u(t)\|_{L^{2}} \rightarrow 0, t \rightarrow \infty$.

\section{Introduction and Main Result}

In this study, we consider the Cauchy problem of the generalized 3D Navier-stokes equations:

$$
\begin{aligned}
u_{t}+(-\Delta)^{\alpha} u+(u \cdot \nabla) u+\nabla \pi & =f, \quad(x, t) \in \mathbb{R}^{3} \times(0, \infty), \\
\nabla \cdot u & =0, \\
u(x, 0) & =u_{0} .
\end{aligned}
$$

Here, $0<\alpha<1$, and $u$ and $\pi$ denote unknown velocity and pressure, respectively. $f$ is the external force and $u_{0}$ is a given initial velocity.

It is well known that when $\alpha=1$, system (1) becomes the classic Navier-Stokes equations. For the Navier-Stokes equations, it is proved that it has a global weak solution

$$
u(x, t) \in L^{\infty}\left(0, T ; L^{2}\right) \cap L^{2}\left(0, T ; H^{1}\right), \quad \forall T>0
$$

for given $u_{0} \in L^{2}\left(\mathbb{R}^{3}\right)$ with $\nabla \cdot u_{0}=0$ [1]. However, the regularity of Leray weak solutions is still an open problem in mathematical fluid mechanics even if much effort has been made [2-4]. It is an interesting problem to investigate the stability properties of the Navier-Stokes equations and related fluid models [5-11]. As regard to the above system (1), the asymptotic stability of weak solution of the generalized 3D Navier-Stokes equation is described as follows. If $u$ is perturbed initially by $\omega_{0}$ without any smallness assumption, then the perturbed system $v$ is governed by the following equations:

$$
\begin{gathered}
v_{t}+(-\Delta)^{\alpha} v+(v \cdot \nabla) v+\nabla \pi=f, \\
\nabla \cdot v=0, \\
v(x, 0)=u_{0}+\omega_{0},
\end{gathered}
$$

where $\omega_{0}$ is the initial perturbation. There is large literature on the stability issue of the classic Navier-Stokes equations and related fluid models [12-17]. The aim of this paper is to show the stability of weak solution in the framework of the homogeneous Besov space. More precisely, with the use of the Littlewood-Paley decomposition and the classic Fourier splitting technique, we can show that when the initial perturbation $\omega_{0} \in L^{2}\left(\mathbb{R}^{3}\right)$, then every weak solution $v(t)$ of the perturbed system (2) converges asymptotically to $u(t)$ as $\|v(t)-u(t)\|_{L^{2}} \rightarrow 0, t \rightarrow \infty$.

Now our result reads as follows.

Theorem 1. Let $f \in L^{2}\left(0, T ; H^{-\alpha}\left(\mathbb{R}^{3}\right)\right), \omega_{0} \in L^{2}\left(\mathbb{R}^{3}\right)$; Suppose that $u(x, t)$ is a weak solution of (1) and that $v(x, t)$ is a weak solution of the perturbed problem (2), respectively. Moreover, if $\nabla u$ also lies in the following regular class:

$$
\nabla u \in L^{p}\left(0, \infty ; B_{q, \infty}^{0}\left(\mathbb{R}^{3}\right)\right), \quad \frac{2 \alpha}{p}+\frac{3}{q}=2 \alpha, \quad 2<q<\infty,
$$

then $\|v(t)-u(t)\|_{L^{2}} \rightarrow 0(t \rightarrow \infty)$. 
The remainder of this paper is organized as follows. In the Section 2, we first recall the Littlewood-Paley decomposition and the Bony decomposition; then we give three key lemmas. And we prove asymptotic stability of the weak solution in the Section 3.

\section{Some Auxiliary Lemmas}

We recall some basic facts about the Littlewood-Paley decomposition (refer to [18]). Let $\delta\left(\mathbb{R}^{3}\right)$ be Schwartz class of rapidly decreasing functions; supposing $f \in \mathcal{S}\left(\mathbb{R}^{3}\right)$, the Fourier transformation $\mathscr{F}$ is defined by

$$
\mathscr{F} f(\xi)=\int_{\mathbb{R}^{3}} e^{-i x \cdot \xi} f(x) d x .
$$

Choose two nonnegative radial functions $\chi, \varphi \in \mathcal{S}\left(\mathbb{R}^{3}\right)$, supported in $\mathscr{B}=\left\{\xi \in \mathbb{R}^{3},|\xi| \leq 4 / 3\right\}$ and $\mathscr{C}=\left\{\xi \in \mathbb{R}^{3}, 3 / 4 \leq\right.$ $|\xi| \leq 8 / 3\}$, respectively, such that

$$
\chi(\xi)+\sum_{j \geq 0} \varphi\left(2^{-j} \xi\right)=1, \quad \xi \in \mathbb{R}^{3} .
$$

Let $h=\mathscr{F}^{-1} \varphi$ and $\widetilde{h}=\mathscr{F}^{-1} \chi$, we define the dyadic blocks as follows:

$$
\begin{aligned}
\Delta_{j} f= & \varphi\left(2^{-j} D\right) f \\
= & 2^{3 j} \int_{\mathbb{R}^{3}} h\left(2^{j} y\right) f(x-y) d y, \quad \text { for } j \geq 0, \\
S_{j} f & =\chi\left(2^{-j} D\right) f=\sum_{-1 \leq k \leq j-1} \Delta_{k} f \\
& =2^{3 j} \int_{\mathbb{R}^{3}} \tilde{h}\left(2^{j} y\right) f(x-y) d y, \\
\Delta_{-1} f & =S_{0} f, \quad \Delta_{j} f=0 \quad \text { for } j \leq-2 .
\end{aligned}
$$

We can easily verify that

$$
\begin{aligned}
\Delta_{j} \Delta_{k} f=\varphi\left(2^{-j} \xi\right) \varphi\left(2^{-k} \xi\right) \hat{f}=0, \quad \text { if }|j-k| \geq 2, \\
\begin{aligned}
\Delta_{j}\left(S_{k-1} f \Delta_{k} f\right)= & \varphi\left(2^{-j} \xi\right) \chi\left(2^{-(k-1)} \xi\right) \widehat{f} \\
& \times \varphi\left(2^{-k} \xi\right) \widehat{f}=0, \quad \text { if }|j-k| \geq 5 .
\end{aligned}
\end{aligned}
$$

Especially for any $f \in L^{2}\left(\mathbb{R}^{3}\right)$, we have the Littlewood-Paley decomposition:

$$
f=S_{0}(f)+\sum_{j \geq 0} \Delta_{j} f, \quad f \in \mathcal{S}^{\prime}\left(\mathbb{R}^{3}\right) .
$$

Now we give the definition of the Besov space. Let $s \in \mathbb{R}$ and $p, q \in[1, \infty]$; the inhomogeneous Besov space $B_{p, q}^{s}\left(\mathbb{R}^{3}\right)$ (see [18]) is defined by the full-dyadic decomposition, such as

$$
B_{p, q}^{s}\left(\mathbb{R}^{3}\right)=\left\{f \in \mathcal{S}^{\prime}\left(\mathbb{R}^{3}\right):\|f\|_{B_{p, q}^{s}}<\infty\right\},
$$

where

$$
\|f\|_{B_{p, q}^{s}}= \begin{cases}\left(\sum_{j=-1}^{\infty} 2^{j s q}\left\|\Delta_{j} f\right\|_{L_{p}}^{q}\right)^{1 / q}, & 1 \leq q<\infty, \\ \sup _{j \geq-1} 2^{j s}\left\|\Delta_{j} f\right\|_{L_{p}}, & q=\infty,\end{cases}
$$

and $\mathcal{S}^{\prime}\left(\mathbb{R}^{3}\right)$ is a dual space of $\mathcal{S}\left(\mathbb{R}^{3}\right)$.

The Bony decomposition (see [19]) will be frequently used; it is followed by

$$
u v=T_{u} v+T_{v} u+R(u, v),
$$

where

$$
T_{u} v=\sum_{j} S_{j-1} u \Delta_{j} v, \quad R(u, v)=\sum_{\left|j^{\prime}-j\right| \leq 1} \Delta_{j} u \Delta_{j^{\prime}} v .
$$

The following Bernstein inequality (see [18]) will be used in the next section.

Lemma 2. Assume that $k, j \in Z$ and $1 \leq p \leq q \leq \infty$, for $f \in \mathcal{S}\left(\mathbb{R}^{3}\right)$, one has

$$
\sup _{|\alpha|=k}\left\|\partial^{\alpha} \Delta_{j} f\right\|_{L^{q}\left(\mathbb{R}^{3}\right)} \leq C 2^{j k+3 j((1 / p)-(1 / q))}\left\|\Delta_{j} f\right\|_{L^{p}\left(\mathbb{R}^{3}\right)}
$$

and the constant $C$ is independent of $j$ and $k$.

In the following, we will introduce two lemmas, which will be employed in the proof of our theorem.

Lemma 3. Suppose that $u, w \in L^{\infty}\left(0, T ; L^{2}\right) \cap L^{2}\left(0, T ; H^{\alpha}\right)$, for all $T>0, \nabla v \in L^{p}\left(0, \infty ; B_{q, \infty}^{0}\right),(2 \alpha / p)+(3 / q)=2 \alpha$, $2<q<\infty$.

Then the trilinear form

$$
F(u, v, w)=\int_{0}^{T} \int_{\mathbb{R}^{3}}(u \cdot \nabla v) w d x d t
$$

is continuous and

$$
\begin{aligned}
|F(u, v, w)| \leq & C\|u\|_{L^{\infty}\left(0, T ; L^{2}\right)}^{1 / p}\|u\|_{L^{2}\left(0, T ; H^{\alpha}\right)}^{1-(1 / p)}\|w\|_{L^{\infty}\left(0, T ; L^{2}\right)}^{1 / p} \\
& \times\|w\|_{L^{2}\left(1, T ; H^{\alpha}\right)}^{1-(1 / p)}\|\nabla v\|_{L^{p}\left(0, T ; B_{q, \infty}^{0}\right)} .
\end{aligned}
$$

In particular, if $u=w$, then

$$
|F(w, v, w)| \leq \frac{1}{2} \int_{0}^{T}\left\|\Lambda^{\alpha} w\right\|_{L^{2}}^{2} d t+C \int_{0}^{T}\|w\|_{L^{2}}^{2}\|\nabla v\|_{B_{q, \infty}^{0}}^{p} d t .
$$


Proof of Lemma 3. We borrow the idea of [20] to prove this lemma. By using of the Littlewood-Paley decomposition and the Bony decomposition, we obtain

$$
\begin{aligned}
F(u, v, w)= & \int_{0}^{T} \int_{\mathbb{R}^{3}}\left(u^{i} w\right) \partial_{i} v d x d t \\
= & \int_{0}^{T} \int_{\mathbb{R}^{3}}\left(T_{u^{i}} w+T_{w} u^{i}+R\left(u^{i}, w\right)\right) \\
& \times\left(\sum_{j} \Delta_{j} \partial_{i} v\right) d x d t \\
= & \sum_{|k-j| \leq 4} \int_{0}^{T} \int_{\mathbb{R}^{3}} S_{k-1} u^{i} \Delta_{k} w \Delta_{j} \partial_{i} v d x d t \\
& +\sum_{|k-j| \leq 4} \int_{0}^{T} \int_{\mathbb{R}^{3}} \Delta_{k} u^{i} S_{k-1} w \Delta_{j} \partial_{i} v d x d t \\
& +\sum_{\left|k-k^{\prime}\right| \leq 1} \sum_{k, k^{\prime} \geq j-3} \int_{0}^{T} \int_{\mathbb{R}^{3}} \Delta_{k} u^{i} \Delta_{k^{\prime}} w \Delta_{j} \partial_{i} v d x d t \\
= & I_{1}+I_{2}+I_{3} .
\end{aligned}
$$

Then we estimate $I_{1}, I_{2}$, and $I_{3}$ one by one. Applying the Hölder inequality and the Bernstein inequality (40), we derive

$$
\begin{gathered}
\left|I_{1}\right| \leq C \sum_{|k-j| \leq 4} \sum_{k^{\prime} \leq k-2} \int_{0}^{T}\left\|\Delta_{k^{\prime}} u^{i}\right\|_{L^{2 q /(q-2)}}\left\|\Delta_{k} w\right\|_{L^{2}}\left\|\Delta_{j} \partial_{i} v\right\|_{L^{q}} d t \\
\leq C \sum_{|k-j| \leq 4} \sum_{k^{\prime} \leq k-2} \int_{0}^{T} 2^{(3 / q) k^{\prime}}\left\|\Delta_{k^{\prime}} u^{i}\right\|_{L^{2}}\left\|\Delta_{k} w\right\|_{L^{2}}\left\|\Delta_{j} \partial_{i} v\right\|_{L^{q}} d t \\
\leq C \sum_{|k-j| \leq 4} \sum_{k^{\prime} \leq k-2} \int_{0}^{T}\left(2^{\left(\alpha / p^{\prime}\right) k^{\prime}}\left\|\Delta_{k^{\prime}} u\right\|_{L^{2}}\right) \\
\times\left(2^{\left(\alpha / p^{\prime}\right) k}\left\|\Delta_{k} w\right\|_{L^{2}}\right) \\
\times\left\|\Delta_{j} \nabla v\right\|_{L^{q}} 2^{\left((3 / q)-\left(\alpha / p^{\prime}\right)\right) k^{\prime}-\left(\alpha / p^{\prime}\right) k} d t
\end{gathered}
$$

where $(1 / p)+\left(1 / p^{\prime}\right)=1$

Since $|k-j| \leq 4, k^{\prime}<k$ and $(2 \alpha / p)+(3 / q)=2 \alpha$ with $2<q<\infty$, then

$$
\begin{aligned}
2^{\left((3 / q)-\left(\alpha / p^{\prime}\right)\right) k^{\prime}-\left(\alpha / p^{\prime}\right) k} & =2^{((3 / q)-\alpha+(\alpha / p)) k^{\prime}-(\alpha-(\alpha / p)) k} \\
& =2^{(3 / 2 q)\left(k^{\prime}-k\right)} \leq C .
\end{aligned}
$$

Thanks to the Sobolev embedding $B_{2, \infty}^{\alpha / p^{\prime}}\left(\mathbb{R}^{3}\right) \hookrightarrow$ $B_{2,2}^{\alpha / p^{\prime}}\left(\mathbb{R}^{3}\right)=H^{\alpha / p^{\prime}}\left(\mathbb{R}^{3}\right)$, we have the following estimate:

$$
\left|I_{1}\right| \leq C \int_{0}^{T}\|u\|_{H^{\alpha / p^{\prime}}}\|w\|_{H^{\alpha / p^{\prime}}}\|\nabla v\|_{B_{q, \infty}^{0}} d t
$$

Similarly, for $I_{2}$, we also have

$$
\left|I_{2}\right| \leq C \int_{0}^{T}\|u\|_{H^{\alpha / p^{\prime}}}\|w\|_{H^{\alpha / p^{\prime}}}\|\nabla v\|_{B_{q, \infty}^{0}} d t
$$

To estimate the last term $I_{3}$, by using the Hölder inequality and the Bernstein inequality we obtain

$$
\begin{gathered}
\left|I_{3}\right| \leq C \sum_{\left|k-k^{\prime}\right| \leq 1} \sum_{k, k^{\prime} \geq j-3} \int_{0}^{T}\left\|\Delta_{k} u^{i}\right\|_{L^{2}}\left\|\Delta_{k^{\prime}} w\right\|_{L^{2}}\left\|\Delta_{j} \partial_{i} v\right\|_{L^{\infty}} d t \\
\leq C \sum_{\left|k-k^{\prime}\right| \leq 1} \sum_{k, k^{\prime} \geq j-3} \int_{0}^{T}\left\|\Delta_{k} u^{i}\right\|_{L^{2}}\left\|\Delta_{k^{\prime}} w\right\|_{L^{2}} \\
\times\left(2^{(3 / q) j}\left\|\Delta_{j} \partial_{i} v\right\|_{L^{q}}\right) d t \\
\leq C \sum_{\left|k-k^{\prime}\right| \leq 1} \sum_{k, k^{\prime} \geq j-3} \int_{0}^{T}\left(2^{\left(\alpha / p^{\prime}\right) k}\left\|\Delta_{k} u\right\|_{L^{2}}\right) \\
\times\left(2^{\left(\alpha / p^{\prime}\right) k^{\prime}}\left\|\Delta_{k^{\prime}} w\right\|_{L^{2}}\right) \\
\times\left\|\Delta_{j} \nabla v\right\|_{L^{q}} 2^{-(3 / q) j-\left(\alpha / p^{\prime}\right)\left(k+k^{\prime}\right)} d t .
\end{gathered}
$$

Since $\left|k-k^{\prime}\right| \leq 1, k, k^{\prime} \geq j-3$ and $(2 \alpha / p)+(3 / q)=2 \alpha, 2<$ $q<\infty$, we have

$$
\begin{gathered}
2^{-(3 / q) j-\left(\alpha / p^{\prime}\right)\left(k+k^{\prime}\right)}=2^{-(3 / q) j-(3 / 2)\left(k+k^{\prime}\right)(1 / q)} \leq 2^{9 / q} \leq C, \\
\left|I_{3}\right| \leq C \int_{0}^{T}\|u\|_{H^{\alpha / p^{\prime}}}\|w\|_{H^{\alpha / p^{\prime}}}\|\nabla v\|_{B_{q, \infty}^{0}} d t .
\end{gathered}
$$

So, we can derive

$$
\begin{aligned}
|F(u, v, w)| & \\
\leq & C \int_{0}^{T}\|u\|_{H^{\alpha / p^{\prime}}}\|w\|_{H^{\alpha / p^{\prime}}}\|\nabla v\|_{B_{q, \infty}^{0}} d t \\
\leq & C\left(\int_{0}^{T}\|u\|_{H^{\alpha / p^{\prime}}}^{2 p^{\prime}} d t\right)^{1 / 2 p^{\prime}}\left(\int_{0}^{T}\|w\|_{H^{\alpha / p^{\prime}}}^{2 p^{\prime}} d t\right)^{1 / 2 p^{\prime}} \\
& \times\left(\int_{0}^{T}\|\nabla v\|_{B_{q, \infty}^{0}}^{p} d t\right)^{1 / p} \\
\leq & C\|u\|_{L^{2 p^{\prime}}\left(0, T ; H^{\alpha / p^{\prime}}\right)}\|w\|_{L^{2 p^{\prime}\left(0, T ; H^{\alpha / p^{\prime}}\right)}}\|\nabla v\|_{L^{p}\left(0, T ; B_{q, \infty}^{0}\right)} .
\end{aligned}
$$

Applying the interpolation inequality, we have

$$
\begin{aligned}
\|u\|_{L^{2 p^{\prime}\left(0, T ; H^{\alpha / p^{\prime}}\right)}} \leq C\|u\|_{L^{\infty}\left(0, T ; L^{2}\right)}^{1-\left(1 / p^{\prime}\right)} \cdot\|u\|_{L^{2}\left(0, T ; H^{\alpha}\right)}^{1 / p^{\prime}} \\
\leq C\|u\|_{L^{\infty}\left(0, T ; L^{2}\right)}^{1 / p} \cdot\|u\|_{L^{2}\left(0, T ; H^{\alpha}\right)}^{1-(1 / p)}
\end{aligned}
$$

Then

$$
\begin{aligned}
|F(u, v, w)| \leq & C\|u\|_{L^{\infty}\left(0, T ; L^{2}\right)}^{1 / p}\|u\|_{L^{2}\left(0, T ; H^{\alpha}\right)}^{1-(1 / p)}\|w\|_{L^{\infty}\left(0, T ; L^{2}\right)}^{1 / p} \\
& \times\|w\|_{L^{2}\left(0, T ; H^{\alpha}\right)}^{1-(1 / p)}\|\nabla v\|_{L^{p}\left(0, T ; B_{q, \infty}^{0}\right)} .
\end{aligned}
$$


Especially if $u=w$, by using the interpolation inequality, we get

$$
\begin{aligned}
|F(u, v, w)| & \leq C \int_{0}^{T}\|w\|_{H^{\alpha / p^{\prime}}}^{2}\|\nabla v\|_{B_{q, \infty}^{0}} d t \\
& \leq C \int_{0}^{T}\|w\|_{L^{2}}^{2\left(1-\left(1 / p^{\prime}\right)\right)}\left\|\Lambda^{\alpha} w\right\|_{L^{2}}^{2 / p^{\prime}}\|\nabla v\|_{B_{q, \infty}^{0}} d t \\
& \leq \frac{1}{2} \int_{0}^{T}\left\|\Lambda^{\alpha} w\right\|_{L^{2}}^{2} d t+C \int_{0}^{T}\|w\|_{L^{2}}^{2}\|\nabla v\|_{B_{q, \infty}^{0}}^{p} d t .
\end{aligned}
$$

Hence, the proof of the lemma is complete.

Let $w(x, t)=v(x, t)-u(x, t)$ denote the difference of $v(x, t)$ and $u(x, t)$, where $u(x, t)$ is a weak solution of $(1)$ and $v(x, t)$ is a weak solution of the perturbed problem (2). Thus $w(x, t)$ satisfies the following equations:

$$
\begin{gathered}
w_{t}+(-\Delta)^{\alpha} w+(v \cdot \nabla) w+(w \cdot \nabla) u+\nabla \pi=0, \\
(x, t) \in \mathbb{R}^{3} \times(0, \infty), \\
\nabla \cdot w=0, \\
w(x, 0)=w_{0} .
\end{gathered}
$$

Lemma 4. Let $w(x, t)$ be the solution of the above problem. Then

$$
|\widehat{w}(\xi, t)| \leq e^{-|\xi|^{2 \alpha} t}\left|\widehat{w}_{0}(\xi)\right|+C|\xi| t .
$$

Proof of Lemma 4. Taking the Fourier transformation of the first equation of (38), we get

$$
\widehat{w}_{t}+|\xi|^{2 \alpha} \widehat{w}=F[-(v \cdot \nabla) w-(w \cdot \nabla) u-\nabla \pi]=: G(\xi, t) .
$$

We can easily obtain

$$
\begin{aligned}
& |F[-(v \cdot \nabla) w]| \leq \sum_{i, j} \int_{\mathbb{R}^{3}}\left|v_{i} w_{j}\right|\left|\xi_{j}\right| d x \leq|\xi|\|v\|_{L^{2}}\|w\|_{L^{2}}, \\
& |F[-(w \cdot \nabla) u]| \leq \sum_{i, j} \int_{\mathbb{R}^{3}}\left|w_{i} u_{j}\right|\left|\xi_{j}\right| d x \leq|\xi|\|w\|_{L^{2}}\|u\|_{L^{2}} .
\end{aligned}
$$

Applying the operator $\nabla \operatorname{div}$ to the first equation of (38), we have

$$
\Delta \pi=\sum_{i, j} \frac{\partial^{2}}{\partial x_{i} \partial x_{j}}\left(-v_{i} w_{j}-w_{i} u_{j}\right),
$$

and taking the Fourier transformation, we get

$$
|\xi|^{2} F[\pi]=\sum_{i, j} \xi_{i} \xi_{j} F\left[-v_{i} w_{j}-w_{i} u_{j}\right] ;
$$

thus

$$
|F[\nabla \pi]| \leq|\xi||F[\pi]| \leq|\xi|\|w\|_{L^{2}}\left(\|u\|_{L^{2}}+\|v\|_{L^{2}}\right) .
$$

Then we have

$$
|G(\xi, t)| \leq|\xi|\|w\|_{L^{2}}\left(\|u\|_{L^{2}}+\|v\|_{L^{2}}\right) .
$$

Thus solving the ordinary differential equation (31) and using (36) gives

$$
\begin{aligned}
|\widehat{w}(\xi, t)| & =\left|\widehat{w}_{0}(\xi) e^{-|\xi|^{2 \alpha} t}+\int_{0}^{t} e^{-|\xi|^{2 \alpha}(t-s)} G(\xi, s) d s\right| \\
& \leq\left|\widehat{w}_{0}(\xi)\right| e^{-|\xi|^{2 \alpha} t}+C|\xi| \int_{0}^{t}\|w\|_{L^{2}}\left(\|u\|_{L^{2}}+\|v\|_{L^{2}}\right) d s \\
& \leq e^{-|\xi|^{2 \alpha} t}\left|\widehat{w}_{0}(\xi)\right|+C|\xi| t,
\end{aligned}
$$

which is the desired assertion of Lemma 4.

\section{Proof of Theorem 1}

The following argument is follows the classic Fourier splitting methods which is first used by Schonbek [21] (see also [22]).

Taking the inner product of the first equation in (38) with $w$ together with the divergence-free condition of $v, w$ we have

$\frac{1}{2} \frac{d}{d t}\|w\|_{L^{2}}^{2}+\int_{\mathbb{R}^{3}}\left|\Lambda^{\alpha} w\right|^{2} d x=-\int_{\mathbb{R}^{3}}(w \cdot \nabla) u \cdot w d x$.

Applying Plancherel's theorem to (38) yields

$$
\begin{gathered}
\frac{1}{2} \frac{d}{d t} \int_{\mathbb{R}^{3}}|\widehat{w}(\xi, t)|^{2} d \xi+\int_{\mathbb{R}^{3}}|\xi|^{2 \alpha}|\widehat{w}(\xi, t)|^{2} d \xi \\
=-\int_{\mathbb{R}^{3}}(w \cdot \nabla) u \cdot w d x .
\end{gathered}
$$

Let $f(t)$ be a continuous function of $t$ with $f(0)=1$, $f(t)>0$ and $f^{\prime}(t)>0$, we can derive the following:

$$
\begin{aligned}
& \frac{d}{d t}\left(f(t) \int_{\mathbb{R}^{3}}|\widehat{w}(\xi, t)|^{2} d \xi\right) \\
& \quad+2 f(t) \int_{\mathbb{R}^{3}}|\xi|^{2 \alpha}|\widehat{w}(\xi, t)|^{2} d \xi \\
& =-2 f(t) \int_{\mathbb{R}^{3}}(w \cdot \nabla) u \cdot w d x \\
& \quad+f^{\prime}(t) \int_{\mathbb{R}^{3}}|\widehat{w}(\xi, t)|^{2} d \xi .
\end{aligned}
$$

By integrating in time from 0 to $t$ for (40), we have

$$
\begin{aligned}
& f(t) \int_{\mathbb{R}^{3}}|\widehat{w}(\xi, t)|^{2} d \xi \\
& \quad+2 \int_{0}^{t} f(s) \int_{\mathbb{R}^{3}}|\xi|^{2 \alpha}|\widehat{w}(\xi, s)|^{2} d \xi d s \\
& =\int_{\mathbb{R}^{3}}\left|\widehat{w}_{0}\right|^{2} d \xi-2 \int_{0}^{t} f(s) \int_{\mathbb{R}^{3}}(w \cdot \nabla) u \cdot w d x d s \\
& \quad+\int_{0}^{t} f^{\prime}(s) \int_{\mathbb{R}^{3}}|\widehat{w}(\xi, s)|^{2} d \xi d s .
\end{aligned}
$$


Noting that $f(t)$ is a scalar function and applying Lemma 3, we get

$$
\begin{aligned}
& \left|\int_{0}^{t} f(s) \int_{\mathbb{R}^{3}}(w \cdot \nabla) u \cdot w d x d s\right| \\
& \leq \frac{1}{2} \int_{0}^{t} f(s)\left\|\Lambda^{\alpha} w\right\|_{L^{2}}^{2} d s+C \int_{0}^{t} f(s)\|w\|_{L^{2}}^{2}\|\nabla u\|_{B_{q, \infty}^{0}}^{p} d s \\
& \leq \frac{1}{2} \int_{0}^{t} f(s) \int_{\mathbb{R}^{3}}|\xi|^{2 \alpha}|\widehat{w}(\xi, s)|^{2} d \xi d t \\
& \quad+C \int_{0}^{t} f(s)\|w\|_{L^{2}}^{2}\|\nabla u\|_{B_{q, \infty}^{0}}^{p} d s .
\end{aligned}
$$

Then,

$$
\begin{aligned}
& f(t) \int_{\mathbb{R}^{3}}|\widehat{w}(\xi, t)|^{2} d \xi \\
& \quad+\int_{0}^{t} f(s) \int_{\mathbb{R}^{3}}|\xi|^{2 \alpha}|\widehat{w}(\xi, s)|^{2} d \xi d s \\
& \leq \int_{\mathbb{R}^{3}}\left|\widehat{w}_{0}\right|^{2} d \xi+\int_{0}^{t} f^{\prime}(s) \int_{\mathbb{R}^{3}}|\widehat{w}(\xi, s)|^{2} d \xi d s \\
& \quad+C \int_{0}^{t} f(s)\|w\|_{L^{2}}^{2}\|\nabla u\|_{B_{q^{0}}^{0}}^{p} d s .
\end{aligned}
$$

Let $B(t)=\left\{\xi \in \mathbb{R}^{3}: f(t)|\xi|^{2 \alpha}<f^{\prime}(t)\right\}$, we have

$f(s) \int_{\mathbb{R}^{3}}|\xi|^{2 \alpha}|\widehat{w}(\xi, s)|^{2} d \xi \geq f^{\prime}(s) \int_{\mathbb{R}^{3}}|\widehat{w}(\xi, s)|^{2} d \xi$

$$
-f^{\prime}(s) \int_{B(s)}|\widehat{w}(\xi, s)|^{2} d \xi
$$

Then,

$$
\begin{aligned}
f(t) & \int_{\mathbb{R}^{3}}|\widehat{w}(\xi, t)|^{2} d \xi \\
\leq & \int_{\mathbb{R}^{3}}\left|\widehat{w}_{0}(\xi)\right|^{2} d \xi+C \int_{0}^{t} f(s)\|w\|_{L^{2}}^{2}\|\nabla u\|_{B_{q, \infty}^{0}}^{p} d s \\
& \quad+\int_{0}^{t} f^{\prime}(s) \int_{B(s)}|\widehat{w}(\xi, s)|^{2} d \xi d s .
\end{aligned}
$$

In addition,

$$
\begin{aligned}
& \int_{0}^{t} f^{\prime}(s) \int_{B(s)}|\widehat{w}(\xi, s)|^{2} d \xi d s \\
& \leq C \int_{0}^{t} f^{\prime}(s) \int_{B(s)}\left(e^{-2|\xi|^{2 \alpha} s}\left|\widehat{w}_{0}(\xi)\right|^{2}+|\xi|^{2} s^{2}\right) d \xi d s \\
& \leq C \int_{0}^{t} f^{\prime}(s)\left(\int_{\mathbb{R}^{3}} e^{-2|\xi|^{2 \alpha}}\left|\widehat{w}_{0}(\xi)\right|^{2} d \xi\right) d s \\
& \quad+C \int_{0}^{t} f^{\prime}(s) s^{2}\left(\frac{f^{\prime}(s)}{f(s)}\right)^{5 / 2 \alpha} d s .
\end{aligned}
$$

Choose $f(t)=(1+t)^{2}$, then

$$
\begin{aligned}
(1+t)^{2} & \int_{\mathbb{R}^{3}}|\widehat{w}(\xi, t)|^{2} d \xi \\
\leq & C+C \int_{0}^{t}(1+s)^{2}\|w\|_{L^{2}}^{2}\|\nabla u\|_{B_{q, \infty}^{0}}^{p} d s \\
& +C \int_{0}^{t}(1+s) \int_{\mathbb{R}^{3}} e^{-2|\xi|^{2 \alpha} s}\left|\widehat{w}_{0}(\xi, s)\right|^{2} d \xi d s \\
& +C(1+t)^{4-(5 / 2 \alpha)}, \\
(1+t)^{2}\|w\|_{L^{2}}^{2} & \\
\leq & C \int_{0}^{t}(1+s) \int_{\mathbb{R}^{3}} e^{-2|\xi|^{2 \alpha} s}\left|\widehat{w}_{0}(\xi)\right|^{2} d \xi d s \\
& +C \int_{0}^{t}(1+s)^{2}\|w\|_{L^{2}}^{2}\|\nabla u\|_{B_{q, \infty}^{0}}^{p} d s \\
& +C(1+t)^{4-(5 / 2 \alpha)} .
\end{aligned}
$$

By using the Gronwall inequality, it follows that

$$
\begin{aligned}
& (1+t)^{2}\|w\|_{L^{2}}^{2} \\
& \leq\left\{C \int_{0}^{t}(1+s) \int_{\mathbb{R}^{3}} e^{-2|\xi|^{2 \alpha} s}\left|\widehat{w}_{0}(\xi)\right|^{2} d \xi d s+C(1+t)^{4-(5 / 2 \alpha)}\right\} \\
& \quad \times \exp \left(\int_{0}^{t}\|\nabla u\|_{B_{q, \infty}^{0}}^{p} d s\right) .
\end{aligned}
$$

Since

$$
\int_{\mathbb{R}^{3}} e^{-2|\xi|^{2 \alpha} t}\left|\widehat{w}_{0}(\xi)\right|^{2} d \xi \leq C(1+t)^{-3 / 2 \alpha} \longrightarrow 0, \quad t \longrightarrow \infty
$$

we derive

$$
\begin{aligned}
&\|w\|_{L^{2}} \leq C(1+t)^{-2} \int_{0}^{t}(1+s) \int_{\mathbb{R}^{3}} e^{-2|\xi|^{2 \alpha}}\left|\widehat{w}_{0}(\xi)\right|^{2} d \xi d s \\
&+C(1+t)^{2-(5 / 2 \alpha)} \longrightarrow 0, \quad t \longrightarrow \infty,
\end{aligned}
$$

which completes the proof of Theorem 1.

\section{Acknowledgments}

The authors want to express their sincere thanks to the editor and the referees for their invaluable comments and suggestions. This work is partially supported by the NNSF of China (11271019), NSF of Anhui Province (11040606M02) and is also financed by the 211 Project of Anhui University (KJTD002B, KJJQ005).

\section{References}

[1] R. Teman, The Navier-Stokes Equations, North-Holland, Amsterdam, The Netherlands, 1977. 
[2] L. Caffarelli, R. Kohn, and L. Nirenberg, "Partial regularity of suitable weak solutions of the Navier-Stokes equations," Communications on Pure and Applied Mathematics, vol. 35, no. 6, pp. 771-831, 1982.

[3] B.-Q. Dong and Z.-M. Chen, "Regularity criteria of weak solutions to the three-dimensional micropolar flows," Journal of Mathematical Physics, vol. 50, no. 10, p. 103525, 13, 2009.

[4] B.-Q. Dong and Z. Zhang, "Global regularity of the 2D micropolar fluid flows with zero angular viscosity," Journal of Differential Equations, vol. 249, no. 1, pp. 200-213, 2010.

[5] P. Constantin and J. Wu, "Behavior of solutions of 2D quasigeostrophic equations," SIAM Journal on Mathematical Analysis, vol. 30, no. 5, pp. 937-948, 1999.

[6] B.-Q. Dong and J. Song, "Global regularity and asymptotic behavior of modified Navier-Stokes equations with fractional dissipation," Discrete and Continuous Dynamical Systems, vol. 32, no. 1, pp. 57-79, 2012.

[7] Z. Q. Luo, "Numerical solution of potential flow equations with a predictor-corrector finite difference method," Journal of Zhejiang University: Science C, vol. 13, no. 5, pp. 393-402, 2012.

[8] Y. Jia, X. Zhang, and B.-Q. Dong, “The asymptotic behavior of solutions to three-dimensional Navier-Stokes equations with nonlinear damping," Nonlinear Analysis, vol. 12, no. 3, pp. 17361747, 2011.

[9] J. Wu, "Generalized MHD equations," Journal of Differential Equations, vol. 195, no. 2, pp. 284-312, 2003.

[10] Z. Q. Luo and Z. M. Chen, "Numerical simulation of standing wave with 3D Predictor-Corrector finite difference method for potential flow equations," Applied Mathematics and Mechanic, vol. 35, no. 8, pp. 931-944, 2013.

[11] J. Wu, "The generalized incompressible Navier-Stokes equations in Besov spaces," Dynamics of Partial Differential Equations, vol. 1, no. 4, pp. 381-400, 2004.

[12] J. Fan and T. Ozawa, "Asymptotic stability for the Navier-Stokes equations," Journal of Evolution Equations, vol. 8, no. 2, pp. 379$389,2008$.

[13] H. Kozono, "Asymptotic stability of large solutions with large perturbation to the Navier-Stokes equations," Journal of Functional Analysis, vol. 176, no. 2, pp. 153-197, 2000.

[14] B.-Q. Dong and Z.-M. Chen, "Asymptotic stability of nonNewtonian flows with large perturbation in $R^{2}$," Applied Mathematics and Computation, vol. 173, no. 1, pp. 243-250, 2006.

[15] B.-Q. Dong and Z.-M. Chen, "Asymptotic stability of the critical and super-critical dissipative quasi-geostrophic equation," Nonlinearity, vol. 19, no. 12, pp. 2919-2928, 2006.

[16] Z.-Q. Luo and Z.-M. Chen, "Sloshing simulation of standing wave with time-independent finite difference method for Euler equations," Applied Mathematics and Mechanics, vol. 32, no. 11, pp. 1475-1488, 2011.

[17] Y. Zhou, "Asymptotic stability for the 3D Navier-Stokes equations," Communications in Partial Differential Equations, vol. 30, no. 1-3, pp. 323-333, 2005.

[18] J.-Y. Chemin, Perfect Incompressible Fluids, Oxford University Press, New York, NY, USA, 1998.

[19] J.-M. Bony, "Calcul symbolique et propagation des singularités pour les équations aux dérivées partielles non linéaires," Annales Scientifiques de l'École Normale Supérieure, vol. 14, no. 2, pp. 209-246, 1981.

[20] B.-Q. Dong and Z.-M. Chen, "On the weak-strong uniqueness of the dissipative surface quasi-geostrophic equation," Nonlinearity, vol. 25, no. 5, pp. 1513-1524, 2012.
[21] M. E. Schonbek, " $L^{2}$ decay for weak solutions of the NavierStokes equations," Archive for Rational Mechanics and Analysis, vol. 88, no. 3, pp. 209-222, 1985.

[22] B. Dong and Y. Li, "Large time behavior to the system of incompressible non-Newtonian fluids in $\mathbb{R}^{2}$," Journal of Mathematical Analysis and Applications, vol. 298, no. 2, pp. 667-676, 2004. 


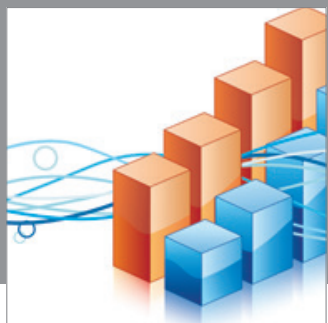

Advances in

Operations Research

mansans

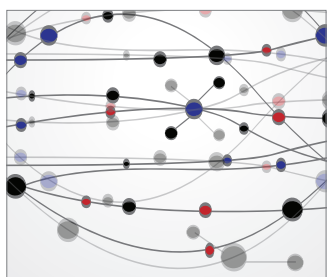

The Scientific World Journal
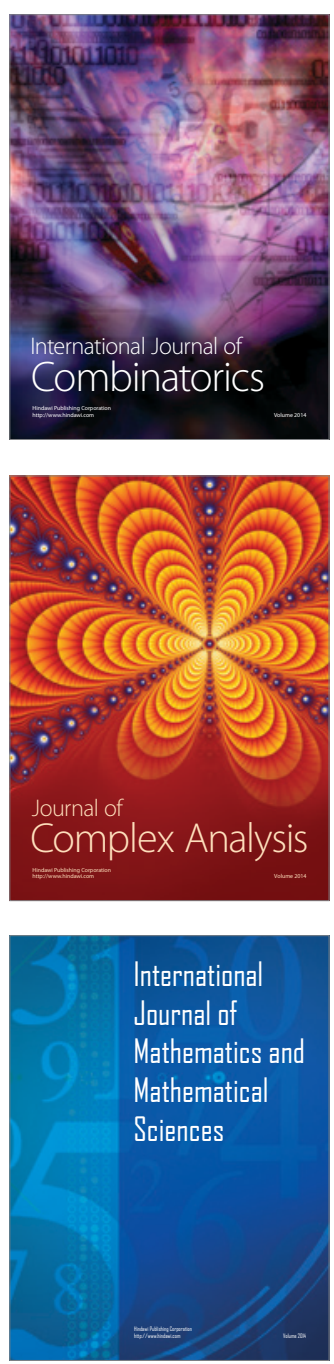
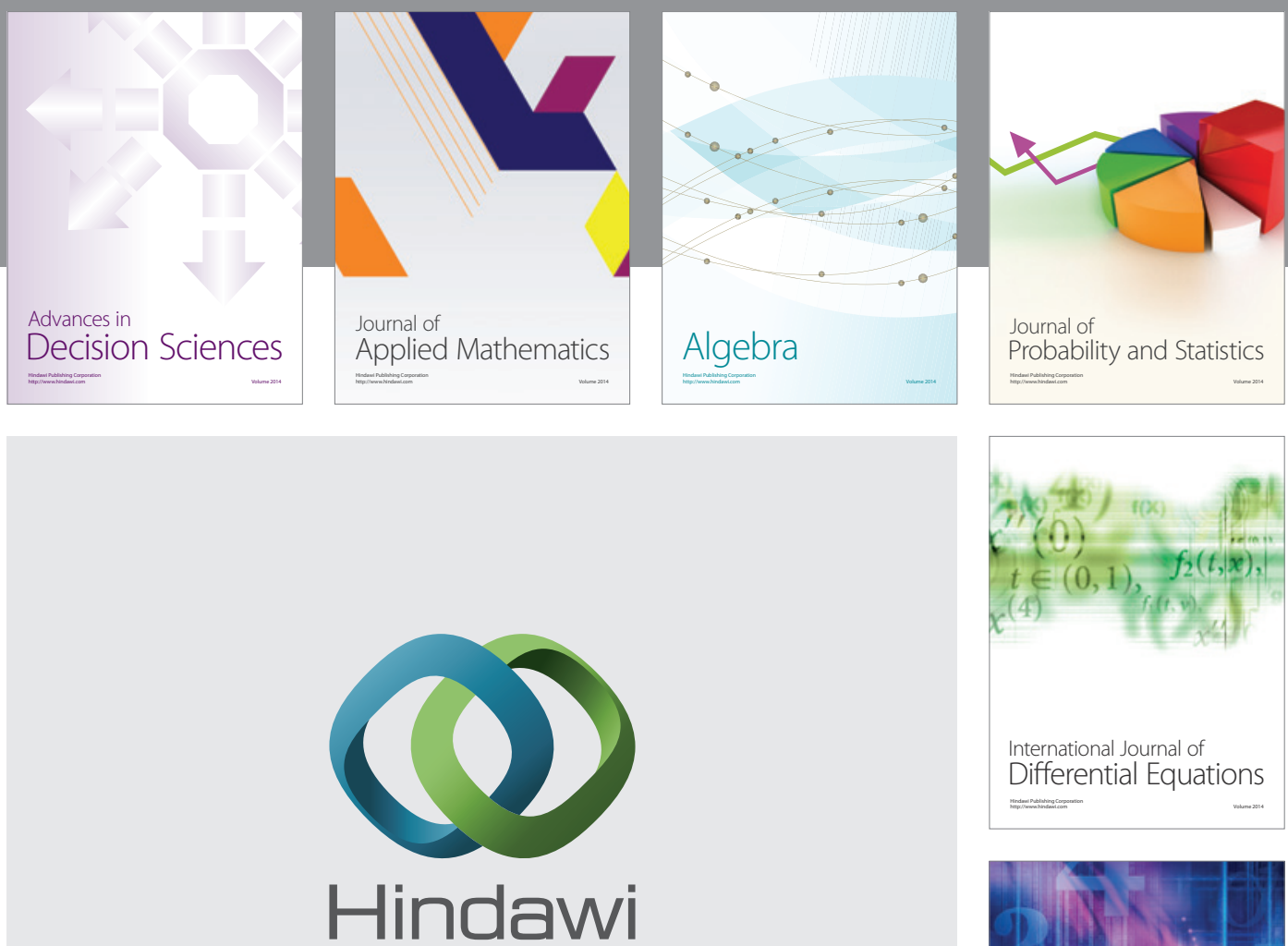

Submit your manuscripts at http://www.hindawi.com
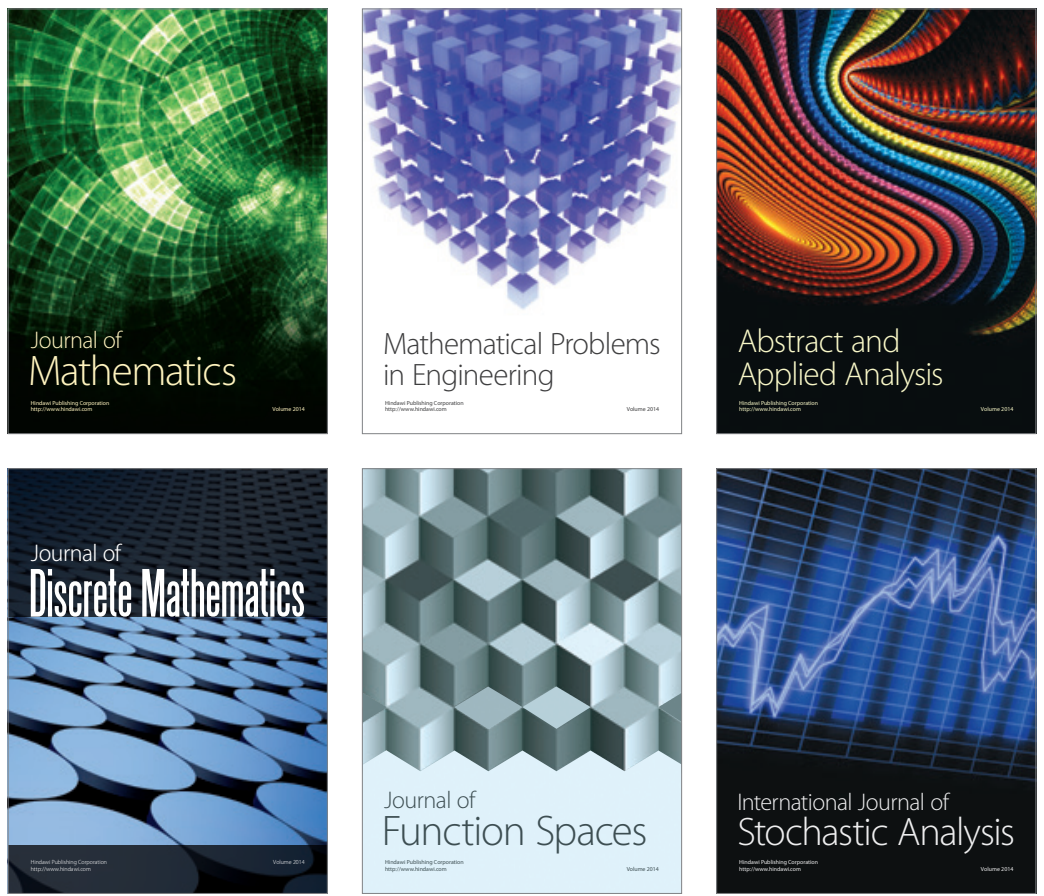

Journal of

Function Spaces

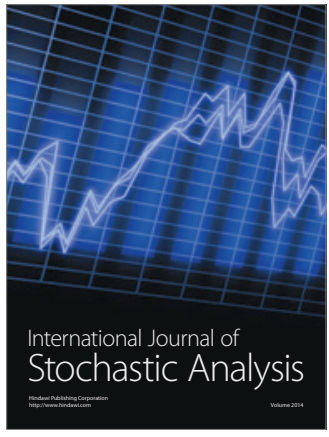

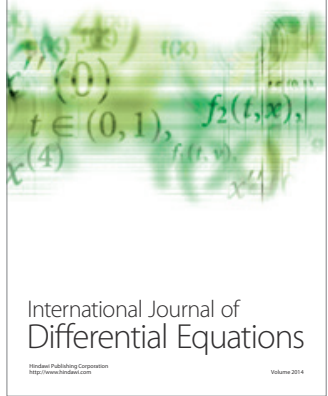
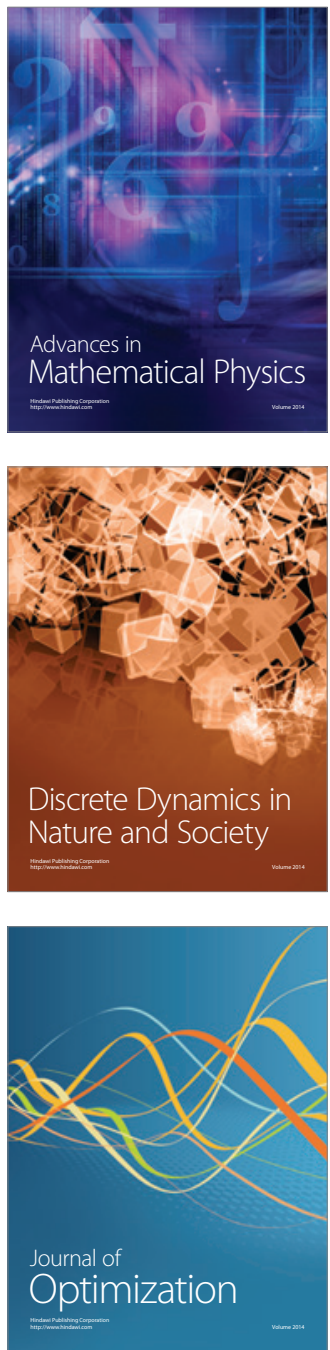